\title{
EXPLORING STRUCTURAL ASPECTS OF NATEGLINIDE POLYMORPHS USING POWDER X-RAY DIFFRACTION
}

\author{
PARNIKA GOYAL, DIMPY RANI, RENU CHADHA* \\ University Institute of Pharmaceutical Sciences, Panjab University, Chandigarh 160014 \\ Email: renukchadha@rediffmail.com
}

Received: 17 Jun 2017 Revised and Accepted: 31 Aug 2017

\begin{abstract}
Objective: The present manuscript highlights the structural aspects of some polymorphic forms of nateglinide using powder $\mathrm{x}$-ray diffraction (PXRD) pattern.

Methods: All the polymorphic forms were isolated as microcrystalline powder, therefore, powder diffraction patterns was used as a tool to determine the crystal structure. For this, Reflux Plus module of BIOVIA Material Studio software was used. Polymorph prediction (PP) and crystal morphology analysis were performed to estimate the global minimum in lattice energy landscape and morphologically important (M. I.) facets, respectively. Besides this, to investigate the behavior of polymorphs in solution phase, in vitro studies (enthalpy of solution, solubility, intrinsic
\end{abstract} dissolution rate) were also performed.

Results: A new form MS was prepared and characterized. The Form H, B, MS and S were found to exist in space group P-1, C2, P-4 and P-42C, respectively. These crystal structures were found to lie on local minima in crystal energy landscape. The stability ranking of nateglinide polymorphs follows the order: Form $\mathrm{MS}<$ Form B<Form $\mathrm{H}<$ Form $\mathrm{S}$.

Conclusion: This research work demonstrates that PXRD is a valuable alternative for determining the structure of microcrystalline powders

Keywords: Nateglinide, Powder diffraction pattern, Crystal structure, Polymorph prediction, Crystal energy landscape, Crystal morphology

(C) 2017 The Authors. Published by Innovare Academic Sciences Pvt Ltd. This is an open access article under the CC BY license (http://creativecommons.org/licenses/by/4.0/) DOI: http://dx.doi.org/10.22159/ijpps.2017v9i10.20795

\section{INTRODUCTION}

The issue of polymorphism is of immense consideration as not only electronic, thermal but also physicochemical [1] (solubility, stability and bioavailability) properties are influenced by this phenomenon. The arrangement of atoms in the crystal lattice is the root cause for molecules to exhibit polymorphism. The identical molecules moiety can be packed into different periodic crystal structure [2] leading to distinct packing arrangement. To understand this arrangement the structure determination is of utmost importance. Single crystal X-rays diffraction (SCXRD) analysis, without any doubt, is the most powerful technique for elucidating the structure of the molecule. But an intrinsic limitation of this technique is the requirement to prepare a crystal of sufficient size, quality and stability. When appropriate single crystals cannot be obtained, PXRD becomes the vital tool to tackle the problem of structure determination [4]. For these reasons, from the past decade, the emphasis was laid on the development, implementation and optimization of new techniques for structure solution from powder diffraction data. Hence, opening up many new opportunities in structural science for microcrystalline powders [4, 5]

The objective of the present manuscript is to use PXRD for studying the structural aspects of polymorphs of nateglinide, a poorly watersoluble oral hypoglycemic agent [6]. Nateglinide has potential to exhibit polymorphism because of the complexity of its structure. The presence of two strong hydrogen bond donors ( $\mathrm{NH}$ and $\mathrm{OH}$ ) and two strong acceptors $(\mathrm{C}=0)$ are responsible for the existence of a variety of intermolecular hydrogen bonding pattern leading to different polymorphic modifications [7]. Many research groups have tried to isolate the polymorphs of nateglinide. The first patent appeared in 1995, explicating the methods for preparing two forms i.e., Form B and $\mathrm{H}$ [8]. Later, a patent unfolded 26 modifications (polymorphs and solvates) of nateglinide, using different solvents, anti-solvents and their mixtures, along with their characterization [9]. Gang Li, et al. with the assistance of PXRD and scanning electronic microscopy (SEM), have reported the existence of new crystal form i.e. Form S [10]. Subsequently, publications by Bruni, et al. reported a new but unstable anhydrous polymorphic modification melting at $102{ }^{\circ} \mathrm{C}$ (form X), which gradually transformed to form $\mathrm{H}$. These researchers have also proposed the method for evaluating the purity of $\mathrm{H}$ polymorph in the binary mixture of $\mathrm{B}$ and $\mathrm{H}$ using diffraction scanning calorimetry (DSC) [11-13]. The monotropic relationship between Form $\mathrm{B}$ and $\mathrm{H}$ and stability co-relation between these two had been established by Upadhyay, et al., using configurational free energy phase diagrams [14]. Recently, Pasha, et al. have evaluated few crystal forms by recrystallizing nateglinide from solvents as well as aqueous solution of surfactants without proper characterization, interpretation and structure determination [15].

The major lacuna in the literature is the unavailability of the structure of either the nateglinide or its reported forms except for the one crystallographic report of its cocrystal with dilute $\mathrm{HCl}$ [8]. Gang $\mathrm{Li}$, et al. has attempted to solve the structure of two polymorphs (Form $\mathrm{H}$ and S) from PXRD pattern using Jade software [16]. However, the arrangement of atoms as well as the hydrogen bond pattern prevailing in different polymorphs was not described. Besides this, Jain, et al. has performed $a b$ initio calculations and semi-empirical calculation on this molecule, reporting few important conformations and concluded that polymorphism in nateglinide might have originated from the dimerization of the molecule [17]

The main focus of the present work is to determine the crystal structure of experimentally crystallized polymorphs using PXRD pattern by BIOVIA Material Studio software. As isolating the perfect crystal for SCXRD is always a tough and skillful task, so was the case with nateglinide. All the forms of nateglinde were isolated only as microcrystalline powder, so powder diffraction patterns were used as a tool to determine the crystal structure.

Experimental hunting of potential polymorphs may be expensive, tedious and time-consuming. Alternate to it or rather an assisting hand to experimental screening is polymorph prediction (PP). All the possible polymorphic forms can be identified by computational crystal structure prediction (CSP), which further calculates their lattice energies. This will aid in spotting polymorphic structure with minimum energy (the global minimum) [18]. The lower energy 
regions in crystal energy landscape grab attention to locate the thermodynamically viable polymorph [19]. All the plausible polymorphs that appear at local minima show different packing arrangement, which subsequently determines its properties. The understanding of crystal structure and the intermolecular forces in crystals are the integral quests to predict, understand or modify the physicochemical properties. These properties are also influenced by the crystal morphology, [20] which is the critical parameter for the quality assessment of the crystalline material [21]. Thus, screening of all the plausible crystal structures and their morphology takes us one step ahead for better understanding and controlling polymorphism [22]. Selection of polymorph with optimum properties is an integral part of formulation and development for crystalline products. Thus this work also deals with in silico prediction of all the possible polymorphs in respective space groups obtained from crystal structure determination along with a simulation of their morphology and thus estimating the M. I. facets. The experimentally prepared polymorphs were also evaluated for their physicochemical properties like solubility, intrinsic dissolution rate (IDR) and enthalpy of solution.

\section{MATERIALS AND METHODS}

\section{Materials}

Nateglinide was obtained as a gift sample from Glenmark Pharmaceuticals Ltd. (Baddi, India). Analytical grade solvents (Sigma Aldrich) were used in this work.

\section{Preparation of polymorphs}

Different polymorphic forms of nateglinide were precipitated out by slow evaporation of solvent-anti-solvent mixture. Form B was obtained by dissolving Form $\mathrm{H}$ (marketed form) in hot ethyl acetate and was subjected to slow evaporation. Apart from this, the same polymorph, Form B was also generated on dissolving Form $\mathrm{H}$ in dioxane (solvent), followed by adding anti-solvent i.e. hexane, heptane and cyclohexane in 1:1 ratio, respectively. Slow evaporation of Form $\mathrm{H}$ from ethanol (solvent): hexane (anti-solvent) (1:3) produced Form MS.

Preparation of Form S was done in two steps. Firstly, Form H was added to heptane (anti-solvent) followed by addition of ethyl acetate (solvent) in ratio 1:1, to solubilize it completely and kept for slow evaporation for $30 \mathrm{~d}$. Then the recrystallized product was heated up to $150{ }^{\circ} \mathrm{C}$ for $30 \mathrm{~min}$ under nitrogen and dried in a desiccator.

\section{Thermal methods of analysis}

DSC thermograms were obtained on Q20, TA Instruments-Waters LLC, USA. The instrument was calibrated for temperature and heat flow accuracy using the melting of pure indium $\left(\mathrm{mp} 156.6^{\circ} \mathrm{C}\right.$ and $\Delta \mathrm{H}$ of $28.45 \mathrm{Jg}-1$ ). A mass between $2-4 \mathrm{mg}$ was taken into the aluminium pan, covered with lid and sealed. DSC curves were obtained under a nitrogen purge of $50 \mathrm{ml}$ per minute at a heating rate of $10^{\circ} \mathrm{C}$ per minute with the temperature range from $50-250^{\circ} \mathrm{C}$.

\section{Powder x-ray diffraction analysis}

The powder diffraction patterns were observed on $\mathrm{x}$-ray diffractometer (XPERT-PRO, PANalytical, Netherlands) using Cu tube anode. The diffractograms were recorded under following conditions: voltage $40 \mathrm{kV}, 35 \mathrm{~mA}$ and fixed divergence slit using the configuration; $2 \theta$ range: $2^{\circ}$ to $50^{\circ}, 0.01$ step size, $10 \mathrm{~s}$ dwell time. Approximately 200 $\mathrm{mg}$ of samples were loaded into the sample holder.

\section{Solid state stability studies}

The physical stability of polymorphs is dependent on atmospheric conditions. It is an important aspect for quality, safety and efficacy of a drug product [23]. To monitor the stability of polymorphs of nateglinide accelerated stability were performed at $40{ }^{\circ} \mathrm{C} / 75 \% \mathrm{RH}$ for 6 mo. Samples were withdrawn after 0,3 and 6 mo and were analyzed with the assistance of DSC and PXRD.

\section{Crystal structure determination from PXRD}

The PXRD pattern was used to solve the crystal structure of polymorphs of nateglinide using the Reflex Plus module of BIOVIA Material Studio software. This process was carried out using Dmol3, X-Cell, Pawley Refinement, Powder Solve and Rietveld Refinement modules [24]. Geometry optimization was done with the gradientcorrected generalized gradient approximation (GGA) and PBE functional in density functional theory (DFT) Dmol3 with double numeric plus polarization (DNP) basis set. It was followed by indexing using X-Cell on 20-35 peaks ranging from $2^{\circ}-30^{\circ} 20$. The unit cell from X-Cell was used as input for Pawley refinement. The weighted rietveld parameter (Rwp) value obtained after the refinement was used to establish the relation between simulated and experimental powder patterns. The optimized structure of the drug molecule and the above-generated unit cell were subjected to Reflex Powder Solve that involved simulated annealing algorithm (10 cycles with 2000100 steps each). Further, rietveld refinement of the structure was performed to obtain a final structure solution.

\section{In silico studies}

\section{a) Polymorph prediction}

All the possible polymorphs of nateglinide in space groups (P-1, C2, P-4, P-42C) were predicted using Polymorph Predictor module of BIOVIA Material studio software. The molecule of nateglinide was geometrically optimized with COMPASS forcefield. The high-level $a b$ initio calculation on these optimized molecules leads to the generation of electrostatic potential (ESP) charges. These were then used as input for polymorph prediction. The same forcefield with Ewald summation for the Van der Waals and electrostatic interactions was used for the calculation and minimization of energy.

The prediction of polymorphs took place in four steps i.e., packing (Monte Carlo simulation), pre-clustering, optimization, and clustering. This results in the generation of the potential crystal structures for the molecule in the selected space group(s). This is followed by grouping the crystals into clusters of similar structures, optimization of generated structures. The optimized structures were then rechecked if any of them converge to the same energy minima. Energy vs density graph (crystal energy landscape) was plotted to search for global minima.

\section{b) Morphology simulation}

Morphology of the polymorphs was simulated using Donnay-Harker rules in the Morphology module of BIOVIA Material Studio, employing COMPASS forcefield. The growth morphology algorithm generates growth faces and their attachment energy (Eatt). This is based on the assumption that the crystal face growth rate is proportional to its attachment energy (AE) [25-27]. The maximum value for the Miller indices and number of growing faces were set to 3 and 200, respectively and inter-planar spacing minima was set to $1.3 \mathrm{~A}^{\circ}$.

\section{Enthalpy of solution}

Enthalpy of the solution was determined by isoperibol solution calorimetry (ISC) (Calorimetry Science Corporation, UTAH, USA) model 4300 in phosphate buffer at $\mathrm{pH} 7.4$ at $37^{\circ} \mathrm{C}$. The procedure followed was same as in our earlier work [28].

\section{Solubility studies}

The solubility studies were done by shaking an excess amount of drug (approx. $5 \mathrm{mg}$ ) in $3 \mathrm{ml}$ phosphate buffer $\mathrm{pH}$ 7.4, in water bath shaker (MSW-275 Macroscientific works, Delhi) at $37^{\circ} \mathrm{C}$ for $24 \mathrm{~h}$ at $200 \mathrm{rpm}$. The resulting slurry was filtered through a $0.45 \mu \mathrm{m}$ membrane filter. The absorbance was measured at $200 \mathrm{~nm}$ with Lambda $25 \mathrm{UV} / \mathrm{VIS}$ spectrometer to determine the concentration of nateglinide.

\section{Intrinsic dissolution studies}

Intrinsic dissolution studies were performed on dissolution test apparatus, DS 8000 (Lab India Analyticals) in phosphate buffer pH 7.4 at $37^{\circ} \mathrm{C}$ with $100 \mathrm{rpm}$ for $3 \mathrm{~h}$. The concentration of nateglinide was determined by measuring absorbance at $200 \mathrm{~nm}$ with Lambda 25 UV/VIS spectrometer.

\section{RESULTS}

\section{Thermal method of analysis}

DSC pattern of a form produced from recrystallization of commercial sample (form $\mathrm{H}$, melting endotherm at $140.98^{\circ} \mathrm{C}$, enthalpy of fusion: $53.88 \mathrm{~J} / \mathrm{g}$ ) from ethyl acetate, dioxane: heptane (1:1), dioxane: 
hexane (1:1), dioxane: cyclohexane (1:1), showed the appearance of single sharp melting endothermic peak at $129.25{ }^{\circ} \mathrm{C}$ (enthalpy of fusion: $23.75 \mathrm{~J} / \mathrm{g}$ ) as shown in fig. 1(a)). The position of the melting endotherm suggested it to be Form $B$, which has already been reported in the literature. However, these authors have used different solvents to prepare Form B $[8,29]$.

A new polymorphically pure form (Form MS) has been isolated by recrystallization from ethanol: hexane (1:3). Its DSC thermogram showed sharp endotherm at $105.12{ }^{\circ} \mathrm{C}$ (enthalpy of fusion: $4.34 \mathrm{~J} / \mathrm{g}$ ) (fig. 1(a)). Recrystallization of Form H from ethyl acetate: heptane (1:1), showed interesting DSC pattern comprising of two endotherms at $129.57^{\circ} \mathrm{C}$ and $174.79^{\circ} \mathrm{C}$, separated by an exotherm at $147.32^{\circ} \mathrm{C}$, (fig. 1 (b))

The thermogram of pure Form S, obtained after heating up to $150^{\circ} \mathrm{C}$, showed a single sharp melting endotherm at $172.36^{\circ} \mathrm{C}$ (enthalpy of fusion: $103.50 \mathrm{~J} / \mathrm{g}$ ) (fig. 1(b)).

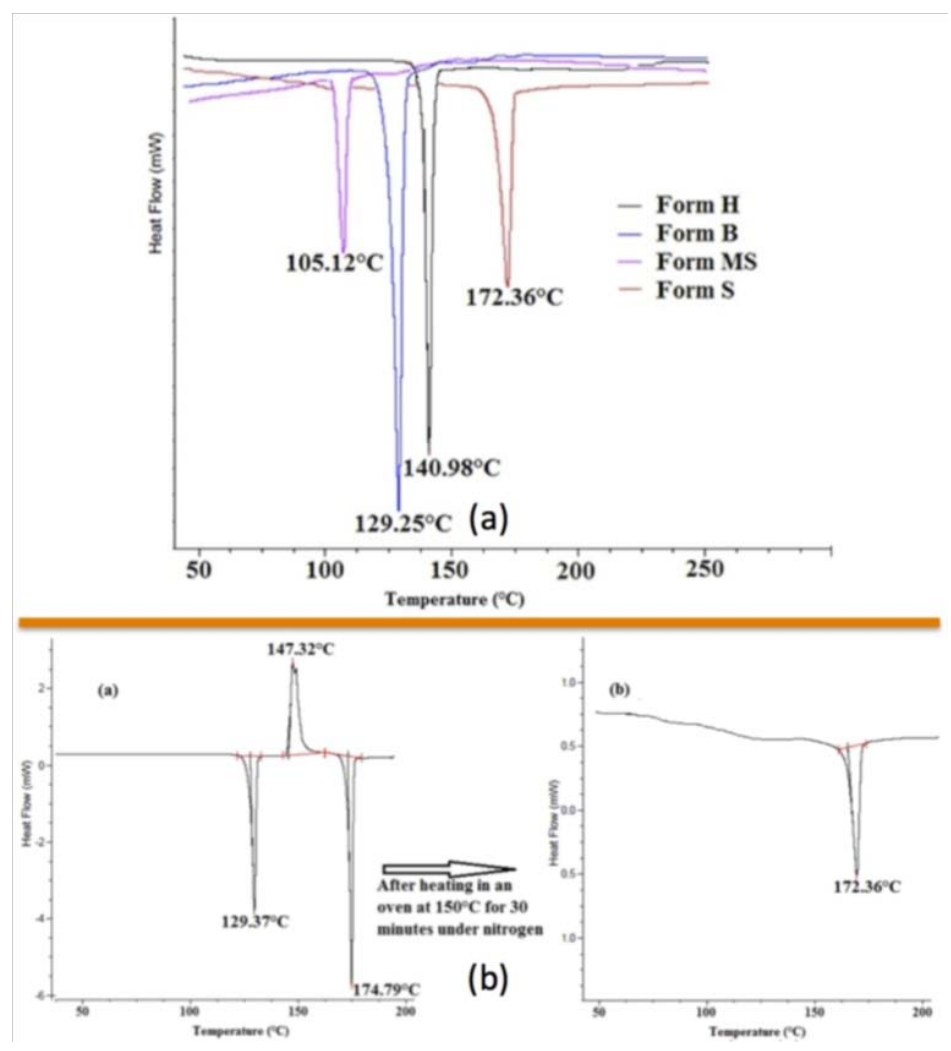

Fig. 1: (a) DSC thermograms of nateglinide polymorphs, (b) DSC thermogram showing isolation of pure Form S
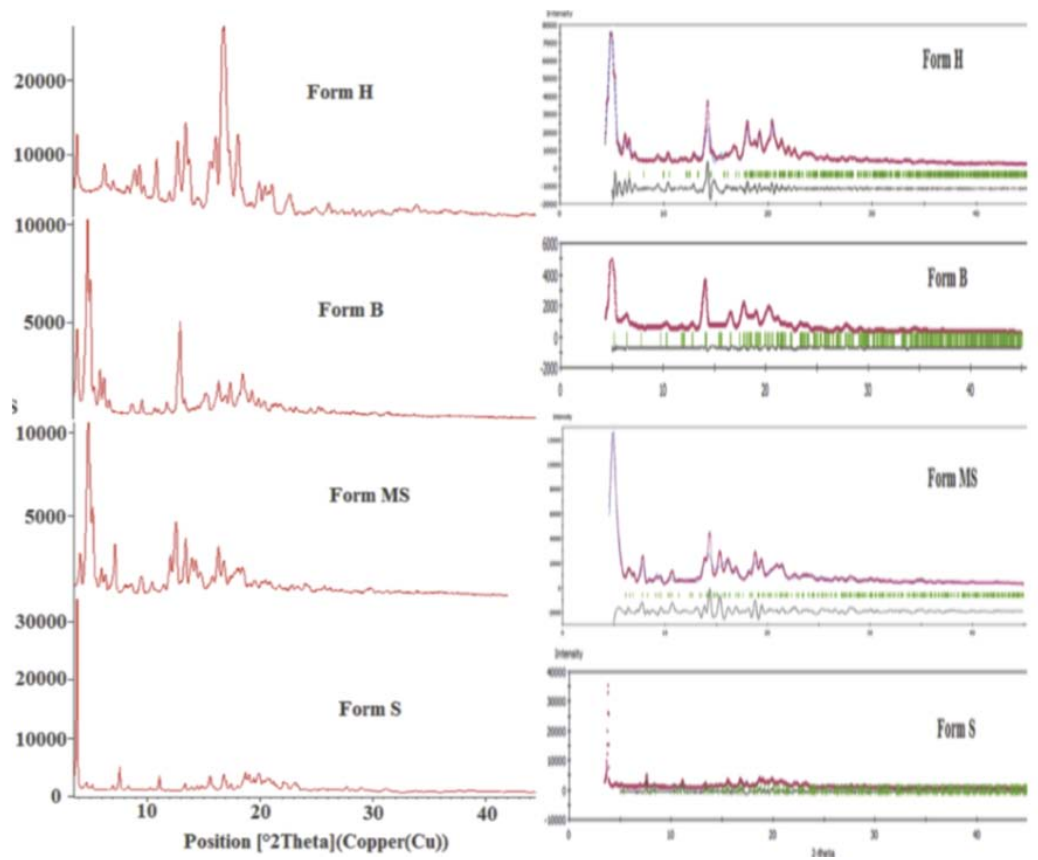

Fig. 2: PXRD pattern of polymorphs of nateglinide and their comparison with the respective simulated pattern (black line indicates the difference between the experimental and simulated PXRD pattern 


\section{Powder x-ray diffraction analysis}

Powder diffraction pattern of prepared polymorphs are given in fig. 2 . The diffraction patterns are different from each other and from the marketed form (Form $\mathrm{H}$ ) as well. The quality of diffraction pattern was adequately good for solving the crystal structure.

\section{Solid state stability studies}

For a drug substance with a number of polymorphs, it is significant to comprehend their relative stability and their inter-conversions. During the stability studies, a consistency in DSC and PXRD pattern of the form $\mathrm{H}, \mathrm{B}$ and S were observed. The DSC and PXRD pattern of all these aged polymorphic forms were found to be same as that of initial samples.

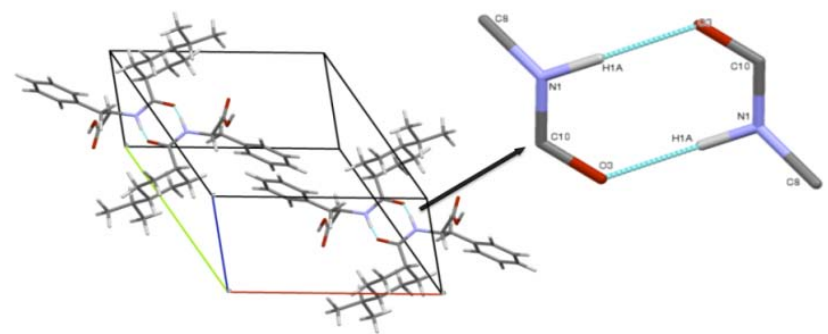

Fig. 3: Packing arrangement along $\mathrm{c}$ axis and homomeric synthon of form $H$

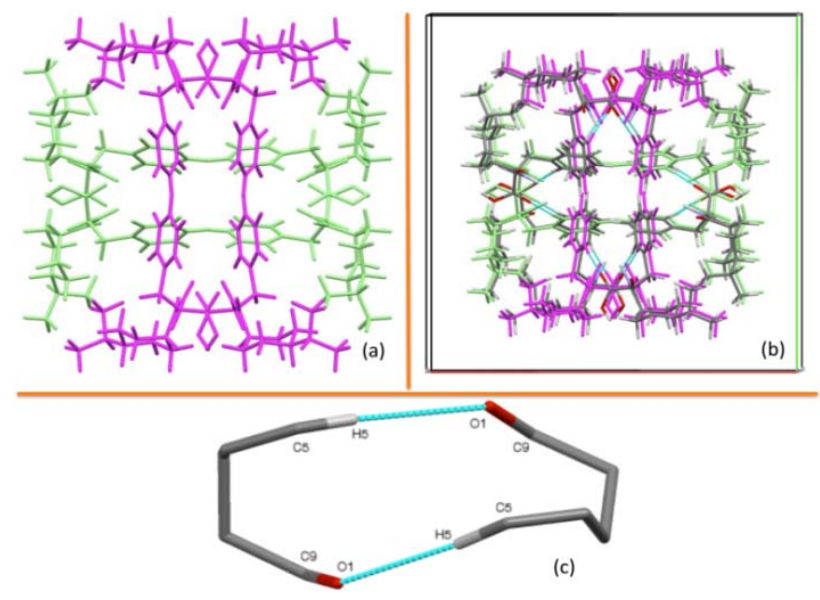

Fig. 4: Form $S$ (a) I-shaped intersecting top view along c axis, (b) packing arrangement, (c) homomeric synthon

\section{Crystal structure determination}

PXRD was used to understand the arrangement of atoms in the crystal lattice, utilizing Reflux Plus module of BIOVIA Material studio. The results obtained were interesting and are unique as shown in fig. 3-6. These results demonstrate the packing arrangement of these polymorphs in the crystal lattice along with their hydrogen bonding pattern. The crystallographic parameters of all the polymorphs are given in table 1 . The crystallographic files are deposited to CCDC with reference number 1401735-1401738. The reliability of determined crystal structures was estimated by the similarity between their experimental and simulated PXRD patterns (comparison shown in fig. 2).

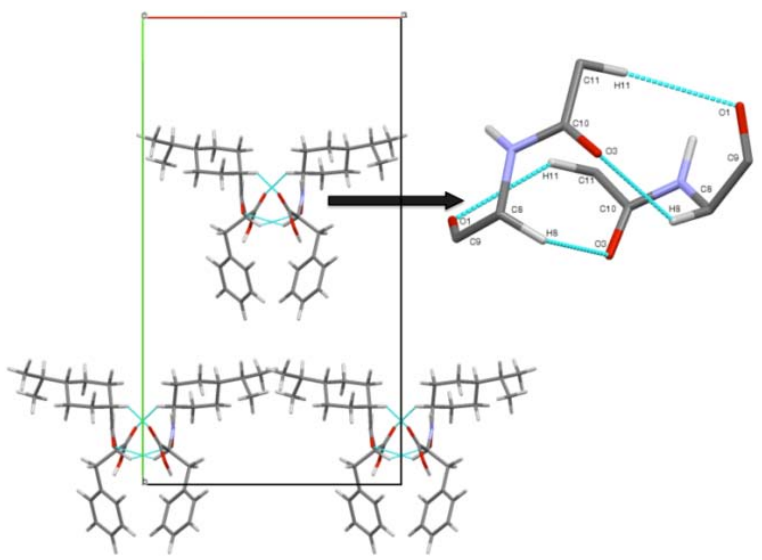

Fig. 5: Packing arrangement along $c$ axis and homomeric synthon of Form B

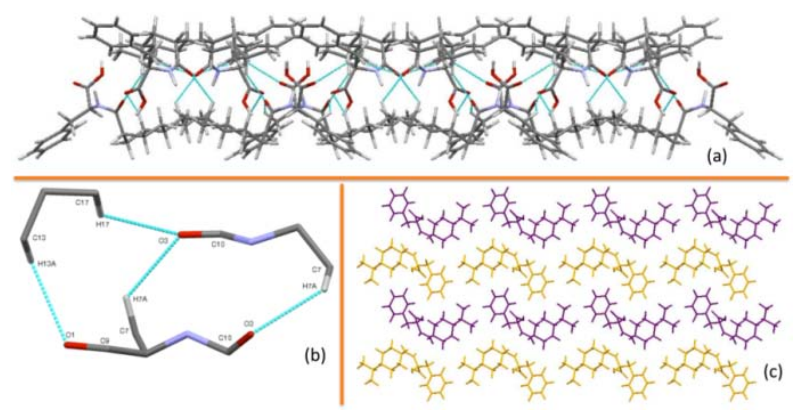

Fig. 6: Form MS (a) sandwiched bilayer along a axis, (b) homomeric synthon, (c) top view along $c$ axis

Table 1: Crystallographic parameters of nateglinide polymorphs

\begin{tabular}{lllll}
\hline Polymorph & Form H & Form B & Form MS \\
\hline Space group(s) & P-1 & C2 & P-4 \\
Crystal Lattice & Triclinic & Monoclinic & Tetragonal \\
a $(\AA)$ & 12.27 & 16.13 & 13.47 & Tetragonal \\
b $(\AA)$ & 11.44 & 27.54 & 13.47 & 23.70 \\
c $(\AA)$ & 8.84 & 18.24 & 41.93 & 23.70 \\
$\alpha($ deg) & 66.46 & 90 & 90 & 12.45 \\
$\beta($ deg) & 104.33 & 108.99 & 90 & 90 \\
$\gamma($ deg) & 116.42 & 90 & 90 & 90 \\
Z & 2 & 4 & 4 & 80 \\
Cell Volume $\left(\AA^{3}\right)$ & 1014.93 & 7659.06 & 7610.04 & 17.77 \\
Rwp $(\%)$ & 14.37 & 5.14 & 6992.19 \\
\hline
\end{tabular}

\section{In silico studies}

Indexing of the powder diffraction pattern of experimentally prepared polymorphs (Form H, B, MS and S) revealed that the forms exists in P-1, C2, P-4 and P-42C, respectively, therefore, the possible crystal structures were explored in these space groups using Polymorph Predictor, employing COMPASS force field.

The search produced more than 2100 distinct hypothetical crystal structures within $30 \mathrm{KJ} / \mathrm{mol}$ of global energy minimum. 
These energy-optimized polymorphs were used as input for CSP calculations, and the resulting crystal energy landscape, comprising energetically feasible crystal structures are shown in fig. 7. The structures corresponding to forms $\mathrm{H}, \mathrm{B}, \mathrm{MS}$ and S were successfully found in the search at local minimum of-55.28,-52.69,-52.28 and$55.40 \mathrm{KJ} / \mathrm{mol}$, respectively.

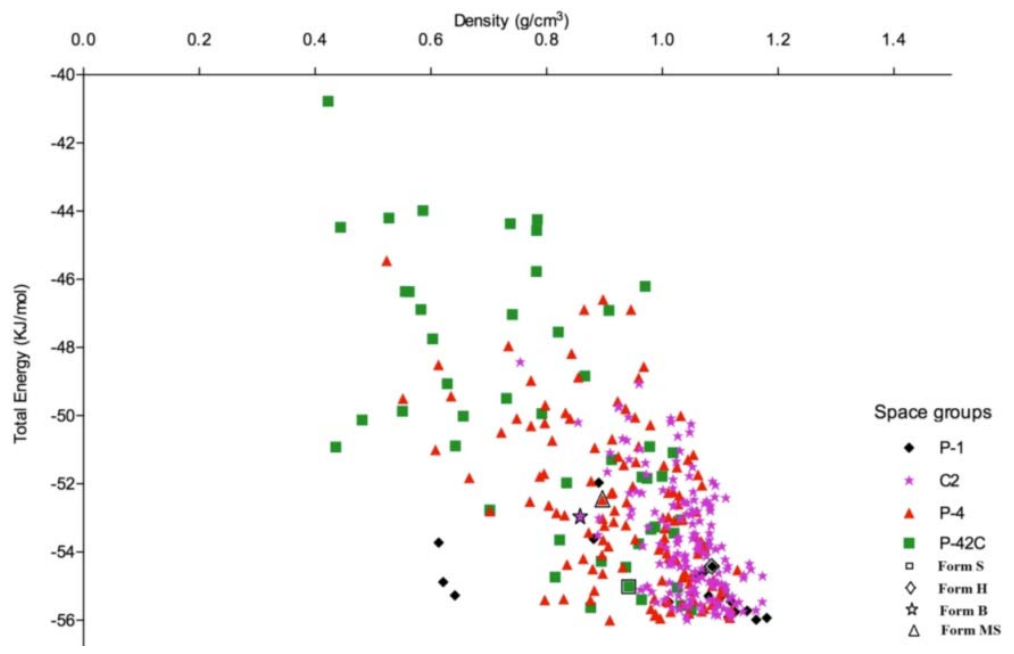

Fig. 7: Crystal energy landscape within $16 \mathrm{KJ} / \mathrm{mol}$ of global energy minimum. The lattice energy minima corresponding to the experimental crystal structure are also shown

Morphology is an important parameter to distinguish various polymorphs, which are in turn affected by the experimental conditions and thus the morphology of nateglinide polymorphs were simulated. The crystal habits along with their M. I. facets (slowest growing faces) of the all forms (H, B, MS and S) are represented in table 2. As the growth rate of a crystal face is relative to its attachment energy, [24] keeping this in mind, morphologies of all the forms were assessed.

Table 2: Crystal morphology prediction

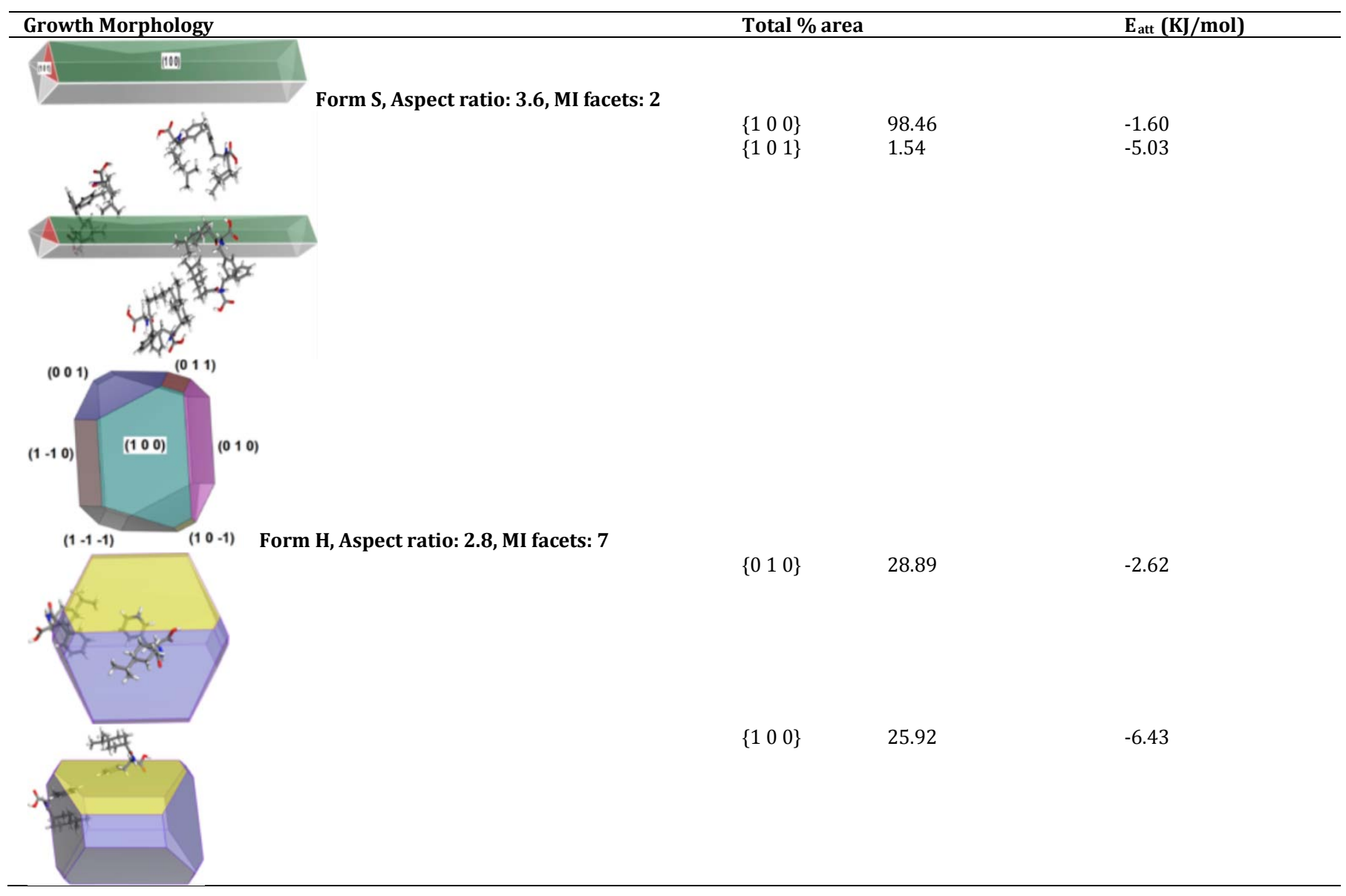




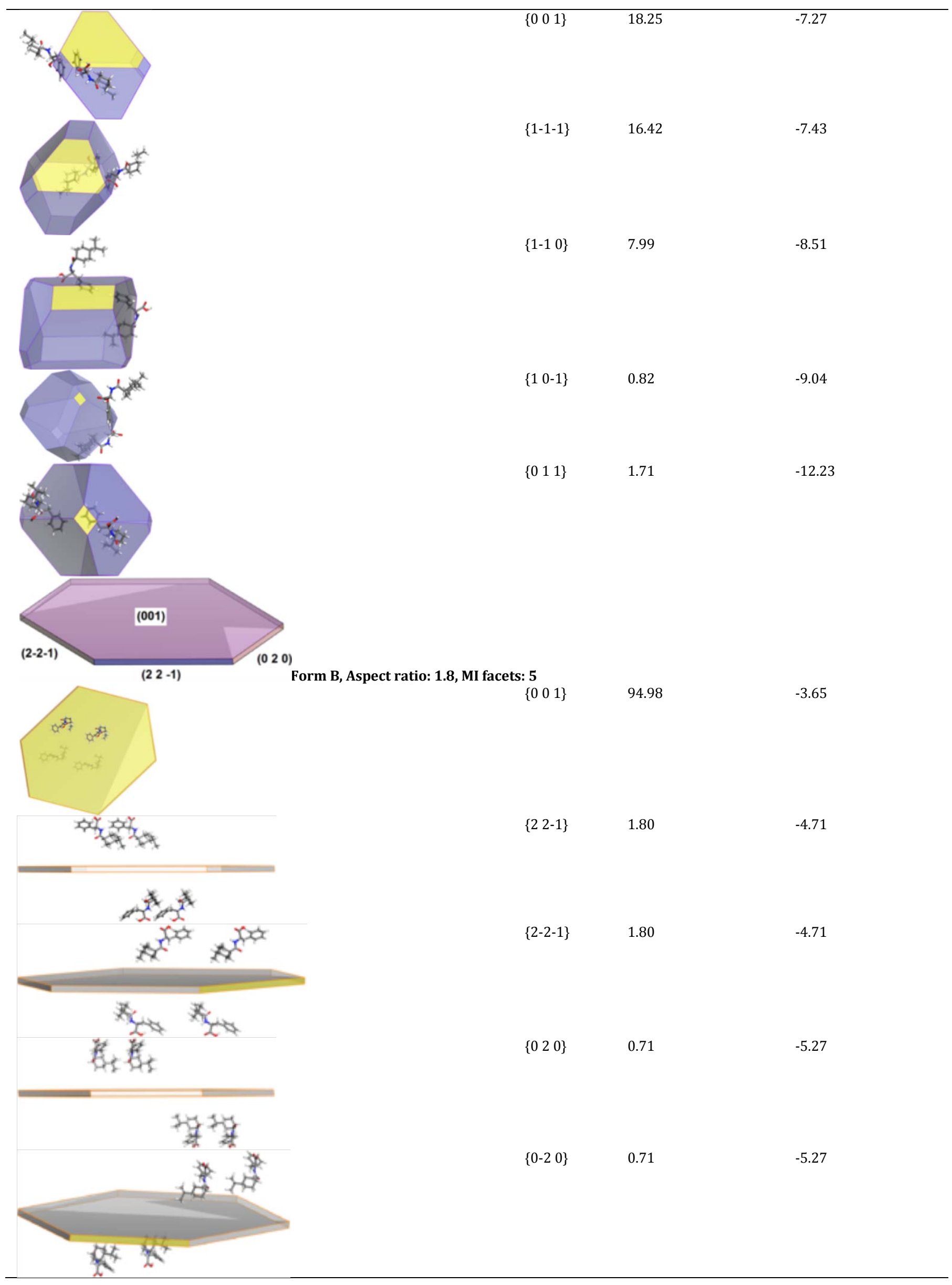




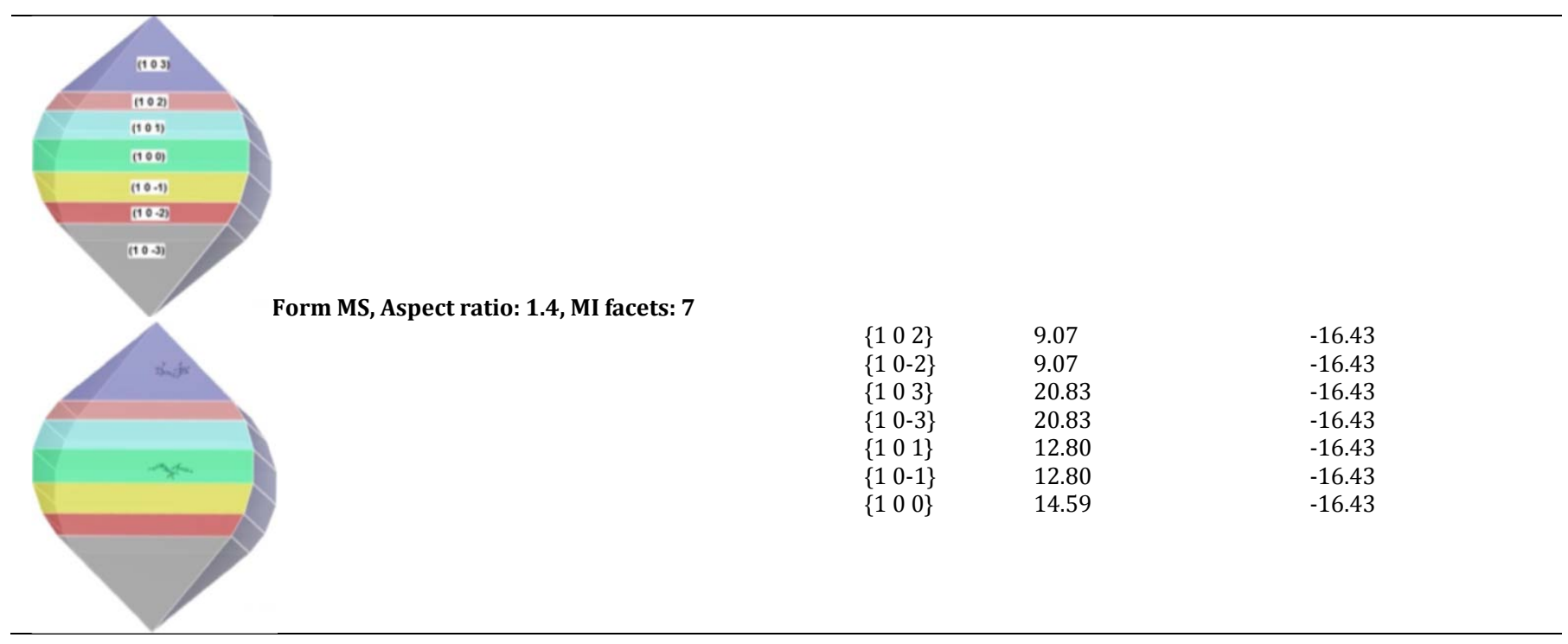

\section{Enthalpy of solution}

The molar enthalpy of solution $\left(\Delta \mathrm{H}_{\mathrm{sol}}\right)$ is an important parameter, which is closely related with the lattice energy and can be used to distinguish different polymorphs. The molar enthalpy of solution $\left(\Delta \mathrm{H}_{\text {sol }}\right)$ of polymorphs was determined in phosphate buffer $\mathrm{pH} 7.4$ at $37{ }^{\circ} \mathrm{C}$. All the polymorphs showed endothermic behaviour in phosphate buffer and the molar enthalpy of solution have been found to be of order: MS form $(1.2 \mathrm{KJ} / \mathrm{mol})<$ Form B $(1.9$ $\mathrm{KJ} / \mathrm{mol})<$ Form $\mathrm{H}(2.2 \mathrm{KJ} / \mathrm{mol})<$ Form S $(4.7 \mathrm{KJ} / \mathrm{mol})$.

\section{Equilibrium solubility and intrinsic dissolution studies}

Equilibrium solubility and IDR of all the polymorphs were determined in phosphate buffer $\mathrm{pH}$ 7.4. The studies suggested that Form MS $\left(89.7 \mathrm{mg} / 100 \mathrm{ml} ; 0.496 \mathrm{mg} / \mathrm{min} / \mathrm{cm}^{2}\right)$ and Form B (87.1 $\mathrm{mg} / 100 \mathrm{ml} ; 0.479 \mathrm{mg} / \mathrm{min} / \mathrm{cm}^{2}$ ) have high solubility and IDR whereas Form $S\left(71.3 \mathrm{mg} / 100 \mathrm{ml} ; 0.263 \mathrm{mg} / \mathrm{min} / \mathrm{cm}^{2}\right)$ has low solubility and IDR, respectively, as compared to the drug (Form H, $86.5 \mathrm{mg} / 100 \mathrm{ml} ; 0.317 \mathrm{mg} / \mathrm{min} / \mathrm{cm}^{2}$ ).

\section{DISCUSSION}

The DSC pattern of Form B shows single endotherm suggesting it to be polymorphically pure. The presence of endotherm at $105.12{ }^{\circ} \mathrm{C}$ indicates the formation of new polymorph, i.e. Form MS. DSC pattern of Form S demonstrates the presence of two endothermic and one exothermic peak. This suggested the conversion of one solid form to another through the molten state. The first endotherm indicates melting of Form B followed by its liquid-solid transition to a stable form, which melts at $174.79{ }^{\circ} \mathrm{C}$ and was similar to the earlier reported Form S. Thermal analysis shows that the Form MS have lowest melting point along with the lowest heat of fusion followed by form B, $\mathrm{H}$ and $\mathrm{S}$ (highest melting point and heat of fusion). So, according to Burger's rule all the forms are monotropically related.

The different diffraction patterns of the prepared polymorphs showed the formation of distinct crystalline phase. The solid state stability studies of polymorphs were carried out. As the thermograms and diffraction patterns of aged samples were similar to that of initial samples, therefore, these samples are found to be stable even after 6 mo. On the other hand, Form MS showed timemediated conversion to Form B after 6 mo. DSC and PXRD pattern of Form MS after 6 mo were found to be similar to that of the Form B, thereby, confirming its conversion to Form B.

The primary objective of this study was to explore the structural aspects of nateglinde polymorphs. To achieve this, the crystal structures of these crystalline polymorphs were determined using PXRD by BIOVIA Material Studio software. Form $\mathrm{H}$ was found to exist in triclinic crystal system, (P-1 space group), which is in concordance with the results reported by Gang et al. [16]. However, the cell parameters are not same. The reason for this may be attributed to the difference in the indexing algorithm. Moreover, they have not described the space group and the packing arrangement. Form $\mathrm{H}$ consists of two molecules in the unit cell (fig. 3). Homomeric synthon between-NH and-CO of an amide group (N1H1 -03) of different molecules is formed. It appears as a dimer (amide-amide dimer), showing hydrogen bonded wavy arrangement of molecules along $\mathrm{c}$ axis in the crystal lattice lying parallel to one another.

The crystal structure of Form $S$ as determined in the present study is tetragonal (P-42C) and the packing arrangement (fig. 4) shows two I-shaped intersecting perpendicular layers consisting of four molecules in each layer. Homomeric synthon (C5-H5 $\cdots 01$ ) between two molecules involving- $\mathrm{CH}$ of the benzene ring and $\mathrm{CO}$ of carboxylic group is formed. They are arranged in such a manner that four molecules appear on anterior and four on posterior face alternatively. The crystal structure of Form $S$ as determined in the preset study is not in agreement with the literature [16]. The comparative profile for both the structures cannot be analyzed, as the procedural details concerning the structural determination from PXRD pattern using Jade software are not provided

The structural aspects of From B and MS have not been explored till date. Form B exists in monoclinic crystal system with C2 space group (fig. 5). Nateglinide molecule is present in in L-shaped conformation forming two homomeric synthons. One homosynthon (03-.H8-C8) is formed between- $\mathrm{CO}$ of amide group and methine- $\mathrm{CH}$ (attached to carboxylic group) of different molecules. The other homosynthon formed (01‥H11-C11), shows the hydrogen bonding between $\mathrm{CO}$ of carboxylic group and-CH of cyclohexyl ring. The packing arrangement is in such a fashion that the benzene ring (head) of two center molecules faces each other whereas the cyclic ring and hydrocarbon chain (tail) are opposite to each other. Other two molecules are present at the corners, with the head emerging out of the unit cell while the tail is approaching each other in the unit cell.

Form MS exists in tetragonal crystal system with P-4 space group (fig. 6). Carbonyl oxygen of amide group bifurcates to form hydrogen bond with methine-CH (attached to cyclohexyl ring) and methylene$\mathrm{CH}$ (attached to benzene ring) to generate $\mathrm{C} 17-\mathrm{H} 17 \cdots \mathrm{O} 3$ and $\mathrm{C} 7-$ $\mathrm{H} 7 \mathrm{~A} \cdots 03$. Another homosynthon $(\mathrm{C} 13-\mathrm{H} 13 \mathrm{~A} \cdots 01)$ is formed by the involvement of- $\mathrm{CO}$ of amide group and-CH of cyclohexyl ring. The molecules are oriented like a rippled bilayer in which all the hydrogen bonds are sandwiched (along a axis). When viewed from c axis, the molecules seem to form zigzag antiparallel layers.

The lattice energy of the polymorphs was estimated by CSP calculations. The crystal energy landscape shows the presence of global minima at- $40.78 \mathrm{KJ} / \mathrm{mol}$. The crystal energy landscape gives explicit account of the packing arrangement rather than the space 
group. The aforesaid landscape was cluttered, indicating the strong tendency to form polymorphs having different motifs, henceforth, packing problem (close packing). The experimental crystal structures lie on local minimum energy rather than global minimum. The reason for this may be attributed to the kinetic inaccessibility of the polymorphs that lie on global minima.

To move one step closer for understanding polymorphism in nategilnide, its morphology was simulated. This demonstrated the M. I. facets along with their attachment energies. The faces with the lowest attachment energies (in terms of magnitude) are the slowest growing and are of more importance [26, 27]. The appearance probability of the observed forms in lattice energy landscape (Form MS> Form B> Form $\mathrm{H}>$ Form S) can be explained easily on the basis of attachment energy [30-32]. Table 2 clearly shows that the magnitude of attachment energy of form MS is-16.43 which is highest of all the forms, and is responsible for its early appearance. The growth rate and attachment energy are inversely related to aspect ratio. Slower the growth rate of $\mathrm{M}$. I. facet, higher will be its aspect ratio implying platelike or needle morphology. The attachment energy and the aspect ratio, in a way, are also related to the stability of the polymorphs. Thus, stability ranking of nateglinide polymorphs follows the order (Form $\mathrm{MS}<$ Form $\mathrm{B}<$ Form $\mathrm{H}<$ Form $\mathrm{S}$ ). The high aspect ratio may also be the reason for the nonexistence of the predicted polymorphs.

The physico-chemical parameters of all the polymorphs were evaluated in phosphate buffer $\mathrm{pH}$ 7.4. The $\Delta \mathrm{H}_{\text {sol }}$ of polymorphs showed endothermic behavior with values different from that reported by Gang Li et al. It is worth mentioning that these authors have used Van't Hoff's equation to calculate the heat of solution from solubility data [33]. The more the magnitude, the higher is the lattice energy, i.e., atoms are held more strongly in crystal lattice. The highest magnitude of $\Delta \mathrm{H}_{\text {sol }}$ of Form $\mathrm{S}$ is indicates that it is associated with the strongest lattice as compared to other forms. Low solubility and low IDR of Form MS implies its strong crystal lattice, which is in concordance with the enthalpy of solution and thermal analysis data.

\section{CONCLUSION}

To understand the arrangement of atoms in crystal lattice, structure determination is of utmost importance. Driven by this objective, the determination of crystal structure from powder diffraction pattern was carried out. This research work demonstrated that PXRD is a valuable alternative for determining the structure of microcrystalline powders. Crystal structures of polymorphs were determined using Reflux Plus module of BIOVIA Material Studio software. The Form H, B, MS and S were found to exist in triclinic, monoclinic, tetragonal and tetragonal, correspondingly. This Form MS is a new isolated polymorphic from. All the possible polymorphs in the respective space groups were explored using Polymorph Predictor. The crystal energy landscape concluded that the experimentally isolated polymorphs lie on local minima. For moving one step ahead in understanding the crystal habit, morphology of these polymorphs was studied. The calculations of attachment energy and aspect ratio from morphological prediction were useful to establish the stability of the polymorphs.

\section{ACKNOWLEDGEMENT}

The authors are greatly thankful to the CSIR, New Delhi (02(0039)/11/EMR-II) and UGC for the financial assistance.

\section{AUTHOR CONTRIBUTION}

These authors contributed equally to this work.

\section{CONFLICT OF INTERESTS}

\section{Declared none}

\section{REFERENCES}

1. Nithya G, Sudha R, Charles CK. Polymorphic behavior of an organic compound using a dynamic thermal and X-ray diffraction technique 2'-chloro-4-methoxy-3-nitro Benzil. Asian J Pharm Clin Res 2017;10:259-62.

2. Butterhof C, Bärwinkel K, Senker J, Breu J. Polymorphism in cocrystals: a metastable form of the ionic co-crystal $2 \mathrm{Hbz} \cdot 1 \mathrm{nabz}$ crystallised by flash evaporation. CrystEngComm 2012;14:6744-9.

3. Harris KDM, Tremayne M, Kariuki BM. Contemporary advances in the use of powder x-ray diffraction for structure determination. Angew Chem Int 2001;40:1626.

4. Harris KDM. New opportunities for structure determination of molecular materials directly from powder diffraction data. Cryst Growth Des 2003;3:887-95.

5. Harris KDM. Crystal structure determination from powder diffraction data. Chem Mater 1996;8:2554-70.

6. Gopi G, Kannan K. Fabrication and in vitro evaluation of nateglinide-loaded ethyl cellulose nanoparticles. Asian J Pharm Clin Res 2015;8:93-6.

7. Tessler L, Goldberg I. Bis(nateglinide) hydronium chloride, and its unique self-assembly into extended polymeric arrays via 0 $\mathrm{H} . . . \mathrm{O}, \mathrm{N}-\mathrm{H} . . . \mathrm{Cl}$ and $\mathrm{O}-\mathrm{H} . . . \mathrm{Cl}$ hydrogen bonds. Acta Crystallogr 2005;61:738-40.

8. Sumikawa M, Koguchi Y, Ohgane T, Irie Y, Takahashi SY. Crystals of $\mathrm{n}$ (trans-4-isopropylcyclohexlycarbonyl)-d phenylalanine and methods for preparing them. US Patent No. 5,463,116; 1995.

9. Ronit Y, Shapiro E, Dolitzky B, Gozlan Y, Gome B. Polymorphic forms of nateglinide. Patent No. 7,148,376 B2; 2006.

10. Gang Li, Qun WX, Xiang YM, Jia YC, Guo QS. Study on stability of nateglinide polymorphism. Chin Chem Lett 2003;14:730-3.

11. Bruni G, Berbennia V, Milanesea C, Girella A, Cardinib A, Viganòb $\mathrm{E}$, et al. Thermodynamic relationships between nateglinide polymorphs. J Pharm Biomed Anal 2009;50:764-70.

12. Brunia G, Berbennia V, Milanesea C, Girella A, Cardinib A Lanfranconib S, et al. New solid modifications of nateglinide. J Pharm Biomed Anal 2010;51:1054-9.

13. Brunia G, Berbennia V, Milanesea C, Girella A, Cardinib A, Lanfranconib $\mathrm{S}$, et al. Determination of the nateglinide polymorphic purity through DSC. J Pharm Biomed Anal 2011;54:1196-9.

14. Upadhyay P, Dantuluri AK, Kumar L, Bansal AK. Estimating relative stability of polymorphs by generation of configurational free energy phase diagram. J Pharm Sci 2012;101:1843-51.

15. Pasha SW, Madhu MVV, Satyanarayana B, Paladugu ND, Rajeev $\mathrm{KM}$, Arief MD, et al. Evaluation of crystal forms of nateglinide. Int J Res Pharm Sci 2013;4:427-37.

16. Gang Li, Jiaying C, Guanglie LU, Qunwei Xu. Determination of the nateglinide polymorphism structure and reduction in the blood glucose level. J Nanjing Normal University 2005;28:65-8.

17. Jain V, Dhaked DK, Kasetti Y, Bharatam PV. Computational study on the conformational preferences in Nateglinide. J Phys Org Chem 2011;25:649-57.

18. Beyer T, Lewis T, Price SL. Which organic crystal structures are predictable by lattice energy minimisation? CrystEngComm 2001;3:178-212.

19. Price SL. From crystal structure prediction to polymorph prediction: interpreting the crystal energy landscape. Phys Chem Chem Phys 2008;10:1996-2009.

20. Moreno-Calvo E, Calvet T, Cuevas-Diarte MA, Aquilano D. Relationship between the crystal structure and morphology of carboxylic acid polymorphs. Predicted and Experimental Morphologies. Cryst Growth Des 2010;10:4262-71.

21. Singh MR, Ramkrishna DA. Comprehensive approach to predicting crystal morphology distributions with population balances. Cryst Growth Des 2013;13:1397-411.

22. Graeme MD. Current approaches to predicting molecular organic crystal structure. Crystallogr Rev 2011;17:3-52.

23. Birva AA, Zarna RD, Ronak RD, Vijayendra Swamy SM, Chetana BP. Stability indicating HPLC method for determination of vilazodone hydrochloride. Int J Curr Pharm Res 2017;9:123-9.

24. Neumann M. X-Cell: a novel indexing algorithm for routine tasks and difficult cases. J Appl Crystallogr 2003;36:356-65.

25. Coombes DS, Catlow CRA, Gale JD, Rohl AL, Price SL. Calculation of attachment energies and relative volume growth rates as an aid to polymorph prediction. Cryst Growth Des 2005;5:879-85.

26. Prywer J. Morphological importance of crystal faces in connection with growth rates and crystallographic structure of crystal. Cryst Growth Des 2002;2:281-6. 
27. Prywer J. Effect of crystal geometry on disappearance of slowgrowing faces. J Cryst Growth 2001;224:134-44.

28. Chadha R, Bhandari S, Arora P, Chhikara R. Characterization, quantification and stability of differently prepared amorphous forms of some oral hypoglycaemic agents. Pharm Dev Tech 2013;18:504-14.

29. Sumikawa M, Makoto M, MIYaZakL K, Shigehro N, Matsuzawa Y. Methods for producing nateglinide B-type crystals. US Patent No. 7,544,834 B2; 2009.

30. Beyer T, Day GM, Price SL. The prediction, morphology, and mechanical properties of the polymorphs of paracetamol. J Am Chem Soc 2001;123:5086-94.

31. Anghel AT, Day GM, Price SL. A study of the known and hypothetical crystal structures of pyridine: why are there four molecules in the asymmetric unit cell? CrystEngComm 2002;4:348-55.

32. Lewis TC, Tocher DA, Day GM, Price SL. A computational and experimental search for polymorphs of parabanic acid-a salutary tale leading to the crystal structure of oxo-ureidoacetic acid methyl ester. CrystEngComm 2003;5:3-9.

33. Gang Li, Qun-Weib LI XU, Cheng R, Huang Yong-Ke, Chang-Gao. Polymorphism and solubility of nateglinide. Acta Chim Sin 2007;65:2817-20.

\section{How to cite this article}

- $\quad$ Parnika Goyal, Dimpy Rani, Renu Chadha. Exploring structural aspects of nateglinide polymorphs using powder X-ray diffraction. Int J Pharm Pharm Sci 2017;9(10):119-127. 